\title{
Cosmology Inspired Design of Biomimetic Tissue Engineering Templates with Gaussian Random Fields
}

\author{
Srinivasan Rajagopalan* and Richard A. Robb \\ Biomedical Imaging Resource, Mayo Clinic College of Medicine, Rochester, MN, USA
}

\begin{abstract}
Tissue engineering integrates the principles of engineering and life sciences toward the design, construction, modification and growth of biological substitutes that restore, maintain, or improve tissue function. The structural integrity and ultimate functionality of such tissue analogs is defined by scaffolds- porous, three-dimensional "trellis-like" structures that, on implantation, provide a viable environment to regenerate damaged tissues. The orthogonal scaffold fabrication methods currently employed can be broadly classified into two categories: (a) conventional, irreproducible, stochastic techniques producing reasonably biomorphic scaffold architecture, and (b) rapidly emerging, repeatable, computer-controlled techniques producing straight edged "contra naturam" scaffold architecture. In this paper, we present the results of the first attempt in an image-based scaffold modeling and optimization strategy that synergistically exploits the orthogonal fabrication techniques to create repeatable, biomorphic scaffolds with optimal scaffold morphology. Motivated by the use of Gaussian random fields (GRF) to model cosmological structure formation, we use appropriately ordered and clipped stacks of GRF to model the three-dimensional pore-solid scaffold labyrinths. Image-based metrology, fabrication and mechanical characterization of these scaffolds reveal the possibility of enabling the previously elusive deployment of promising benchside tissue analogs to the clinical bedside.
\end{abstract}

\section{Introduction}

Tissue and organ loss and defects occur in a wide variety of clinical situations, and their reconstruction to restore structural and functional integrity is a challenging but necessary step in the patient's rehabilitation. Tissue engineering attempts to address this challenge by regenerating damaged or malfunctioning organs from the recipient's own cells. The cells are harvested and embedded on a natural or synthetic carrier material, template or scaffold, that is ultimately implanted at the desired site. On implantation and integration, blood vessels attach to the new tissue, the scaffold dissolves, and the newly grown tissue clings, crawls, proliferates and eventually blends in with its surroundings. The scaffold architecture plays the essential and crucial role in defining the structural integrity and ultimate functionality of the engineered tissues.

Tissue engineering scaffolds are conventionally fabricated using "particulate leaching" [1]- a stochastic approach in which water soluble porogen, such as salt,

\footnotetext{
Is currently with GE Global Research, Bangalore, India.
} 
sugar, or gelatin particles are included into biocompatible material before polymerization, and are subsequently leached out. The footprints left by the leached out porogen forms the pores within the material. Manual intervention, inconsistent and inflexible processing procedures, unpredictable morphology, anti-biomorphic crystalline footprints of the porogens and irreproducibility are critical limitations of this technique. Alternatively, scaffolds can be fabricated reproducibly using computer-controlled rapid prototyping devices. While such techniques offer unique ways to precisely control scaffold architecture, the CAD-based scaffold models, with exception of [2], employed in these approaches have straight edges and sharp turns. Such partitions do not provide the biomorphic environment suitable for cell attachment, migration and proliferation [3]. Figure 1 shows representative $\mu$ CT cross sections of the scaffolds fabricated with the stochastic and deterministic techniques.
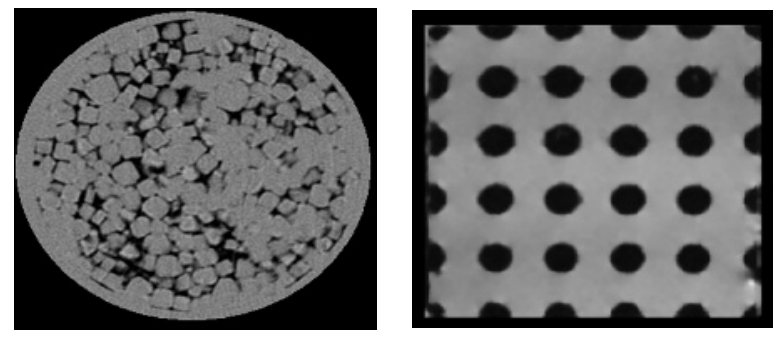

Fig. 1. Representative $\mu \mathrm{CT}$ cross sections of tissue engineering scaffolds fabricated with stochastic (left) and deterministic (right) processes

Towards synergistically exploiting the advantages of these orthogonal techniques, we propose the use of rapid prototyping devices to deterministically fabricate the pore-solid labyrinths designed using stochastic processes. Instead of letting the driven-by-nature reaction-diffusion random processes determine the ultimate suboptimal scaffold structure, we use computer-generated Gaussian random fields (GRF) as "ab initio" models for subsequent optimization driven by the structural requirements for functional scaffolds.

The inspiration for using GRF as tissue engineering templates is drawn from its successful use as the primordial density fluctuation model to explain the complex hierarchy of nodes, filaments and sheets interlocking large voids forming the largescale cosmological structures [4,5]. This success, achieved at the mega-structural level, when translated and perfected with optimized design and controlled fabrication techniques at the macro- and microscopic lamellar, architectural and whole organ levels, could finally realize the significant potential of the previously elusive biomorphic tissue analogs.

\section{Gaussian Random Fields: Theory and Generation}

A stochastic process is a collection of random variables $\{Z(\mathbf{s}): \mathbf{s} \in \mathrm{D}\}$ indexed over a set $\mathrm{D}$. A random field is a stochastic process where $\mathrm{D} \subseteq \mathfrak{R}^{2}$. In GRF, the finite 
collections $\left(\mathrm{Z}\left(\mathrm{s}_{1}\right), \ldots \mathrm{Z}\left(\mathrm{s}_{\mathrm{n}}\right)\right)$ are jointly normal. The distribution of such a field is completely determined by its mean and covariance functions which describe the spatial trend and spatial association respectively [6]. While any mean function $m$ could be used, the covariance function $r$ should be symmetric and positive definite. Following are some of the popular $r(l)$ ( $l$ is the distance between points where the covariance is computed) covariance functions used to model GRF :

$$
\text { Matern correlation }\left(\mathrm{C}_{\mathrm{M}}\right)[7]: r(l)=\frac{1}{2^{\theta_{2}-1} \Gamma\left(\theta_{2}\right)}\left(\frac{l}{\theta_{1}}\right) \kappa_{\theta_{2}}\left(\frac{l}{\theta_{1}}\right), l \neq 0 ;=1, l=0 \text {. }
$$

where $\theta_{1}, \theta_{2}>0, \Gamma$ is the gamma function and $\kappa_{\theta 2}$ is a modified Bessel function of the third kind of order $\theta_{2}$.

Spherical correlation $\left(\mathrm{C}_{\mathrm{S}}\right)[8]: r(l)=1-\frac{3}{2}\left(\frac{l}{\theta}\right)+\frac{1}{2}\left(\frac{l}{\theta}\right)^{3}, l \leq 0 ;=0, l>0$. where $\theta>0$

Exponential correlation $\left(\mathrm{C}_{\mathrm{E}}\right): r(l)=\theta_{1}^{l^{\theta_{2}}}$, where $\theta_{1} \in(0,1)$ and $\theta_{2} \in(0,2]$.

Rational quadratic correlation $\left(\mathrm{C}_{\mathrm{R}}\right)[9]: r(l)=\left(1+\frac{l^{2}}{\theta_{1}^{2}}\right)^{-\theta_{2}}$, where $\theta_{1}, \theta_{2}>0$.

The statistical characteristics of these covariance functions are discussed in [10]. In addition to their mathematical elegance and ubiquitous relevance, GRF adequately describe the morphology of bicontinuous microemulsions, polymer blends and foams [11-13]. This provides us the impetus to explore the use of GRF in tissue engineering.

To generate a zero-mean GRF $\mathbf{Z}(s)$ with a positive-definite covariance function $r$ in a finite subset $\mathrm{S}=\left\{\mathrm{s}_{1}, \ldots, \mathrm{s}_{\mathrm{n}}\right\} \subset \mathfrak{R}^{2}$, a $n \times n$ symmetric positive-definite covariance matrix $\mathbf{T}$ such that $\mathrm{T}_{\mathrm{ij}}=\operatorname{Cov}\left(\mathrm{Z}\left(\mathrm{s}_{\mathrm{i}}\right), \mathrm{Z}\left(\mathrm{s}_{\mathrm{j}}\right)\right)=r\left(\mathrm{~s}_{\mathrm{i}}, \mathrm{s}_{\mathrm{j}}\right)$ is constructed from which the multivariate normal vector $\left(\mathrm{Z}\left(\mathrm{s}_{1}\right), \ldots \mathrm{Z}\left(\mathrm{s}_{\mathrm{n}}\right)\right) \sim N(0,1)$ is generated using classical Cholesky decomposition [14]. Other computationally efficient methods to generate GRF over large grids are addressed in [10]. Figure 2 shows a mosaic of GRF generated with the different covariance functions described above. For this study, we generated 50 GRF (grid size 256 x 256) for each of the covariance functions. The inplane resolution was assumed to be 100 microns.

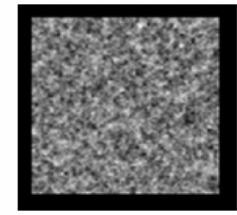

(a) $\mathrm{C}_{\mathrm{M}}\left(\theta_{1}, \theta_{2}\right)=(\mathbf{1 , 1})$

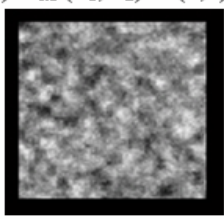

(d) $\mathrm{C}_{\mathbf{S}}(\boldsymbol{\theta})=(\mathbf{1 0})$

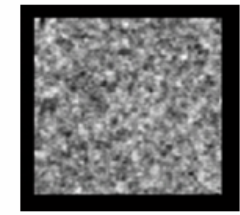

(b) $\mathrm{C}_{\mathbf{M}}\left(\theta_{1}, \theta_{2}\right)=(\mathbf{1}, 2)$

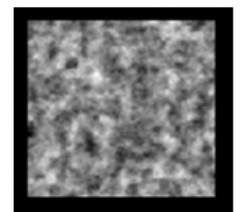

(e) $C_{E}\left(\Theta_{1}, \theta_{2}\right)=(0.9,1.5)$

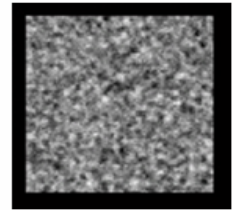

(c) $\mathrm{Cs}_{\mathrm{s}}(\theta)=(5)$

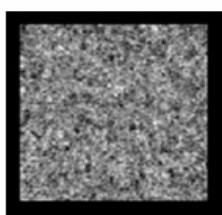

(f) $C_{R}\left(\theta_{1}, \theta_{2}\right)=(\mathbf{1 . 1}, 1.1)$

Fig. 2. Representative zero-mean GRF generated with different covariance functions $\mathrm{C}_{(.)}$. The parameters $\theta_{1}, \theta_{2}$ and $\theta$ used for each $C_{(.)}$are specified under the respective images. 


\section{GRF-Based Tissue Engineering Scaffold Modeling}

Volumetric images for stochastic tissue engineering scaffolds can be obtained by stacking multiple GRF which represent the cross sectional images. The biphasic poresolid labyrinths can be modeled from these stacks by clipping the GRF at appropriate levels. Figure 3 shows the result of clipping the GRFs in Figure 2 at a level of 0.6 which, due to the normal distribution of the GRF, directly translates to an in-plane porosity (void fraction) of $60 \%$. Figure 3 shows that clipped GRFs naturally possess macro and microporous networks- an essential requirement in tissue engineering scaffolds for concomitant cell proliferation and vascular infiltration.

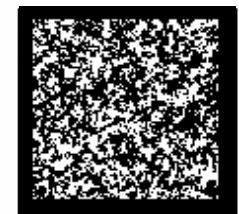

(a) $C_{M}\left(\theta_{1}, \theta_{2}\right)=(1,1)$

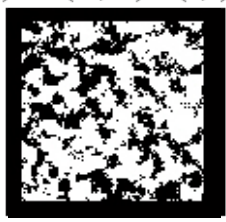

(d) $\mathrm{C}_{\mathbf{S}}(\theta)=(10)$

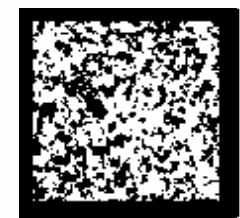

(b) $\mathrm{C}_{\mathrm{M}}\left(\theta_{1}, \theta_{2}\right)=(\mathbf{1}, 2)$

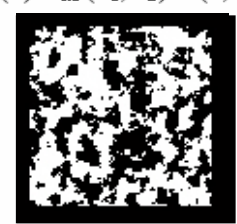

(e) $C_{E}\left(\theta_{1}, \theta_{2}\right)=(0.9,1.5)$

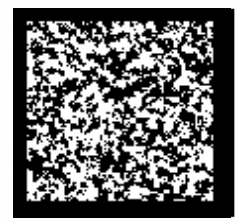

(c) $\mathrm{Cs}_{\mathrm{S}}(\theta)=(5)$

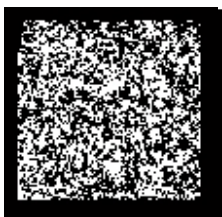

(f) $C_{R}\left(\theta_{1}, \theta_{2}\right)=(1.1,1.1)$

Fig. 3. Clipping GRF (Figure 2) at a level of $\alpha$ yields binary random pore-solid sections with an in-plane porosity of $\alpha^{*} 100 \%$. In this example, $\alpha=0.6$

The binary random fields generated with the desired in-plane porosity can be stacked together to form the three-dimensional pore-solid labyrinths. However, the randomness of the individual GRF makes the brute-force stacking sub optimal. Optimal scaffold architecture should be adequately porous with least tortuous and globally interconnected pore network [1]. Additionally, within micron ranges, the scaffold should have reasonably smooth out-of-plane spatial gradients so that the cytofilaments of proliferating cells can anchor strongly to the substrate [3].

Global porosity, tortuosity and interconnectivity can be maximized by permuting the clipped GRF such that the binary similarity between adjacent sections is maximized. In [16] an exhaustive list of binary similarity measures has been analyzed. Any of the symmetric measures explored therein can be used in the permutation; in this study we have used the Dice similarity coefficient [17]. By permuting the GRF sections based on maximization of adjacent section similarity, the out-of-plane overlap of pore and solid subspaces are maximized. This optimization decreases pore tortuosity, increases global pore connectivity and simultaneously improves the scaffold's structural integrity and mechanical strength.

To provide the appropriate out-of-plane spatial gradients, the permuted cross sections are assumed to be separated by an interstice (we chose an interstitial distance 
of 500 microns). Shape based interpolation within the interstice facilitates the smooth morphing of the pore boundaries. We employed a robust shape-based interpolation technique [18] to generate intermediate cross sections at 100 microns apart. Briefly, the technique uses a feature-guided approach to interpolate porous and tortuous binary objects. The feature points derived from the boundaries of the candidate source objects are matched non-linearly. The intermediate objects are obtained by appropriately blending the warped source objects. A robust outlier-rejecting, nonlinear point matching algorithm based on thin-plate splines is used for establishing the feature correspondence. This scheme correctly handles objects with holes, large offsets and drastic invaginations. The mosaic in figure 4 shows a representative set of consecutive cross sections obtained by interpolating the appropriately ordered clipped GRF. In contrast to the straight edged crystalline footprints (Figure 1) reminiscent in conventional stochastic scaffolds, GRF-based scaffolds have curved partitions which favor tissue ingrowth [3]. Cell proliferation is further enhanced by the smooth out-ofplane spatial gradients.
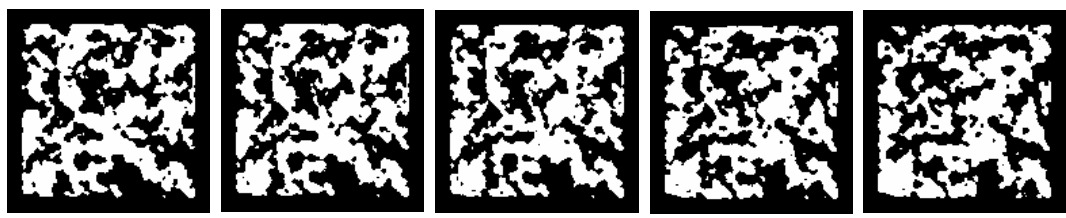

Fig. 4. Representative intermediate sections of the porous scaffolds generated by clipping GRF with covariance function $\mathrm{C}_{\mathrm{E}}\left(\theta_{1}, \theta_{2}\right)=(0.9,1.5)$

\section{Image Based Metrology of GRF-Scaffolds}

The regeneration of specific tissues guided by tissue analogues is dependent on diverse architectural indices like pore size, porosity, pore shape, pore interconnectivity, tortuosity and scaffold stiffness [19]. These indices can be computed non-destructively with the binary pore-solid subspace. While bulk averaged quantities such as porosity and surface area are derived directly from the pore-solid delineations, the spatially distributed geometric indices are derived from the medial axis representations of the pore network.

The computational framework for image-based metrology of porous scaffolds and the biological relevance of the computed indices are described in [20]. We provide succinct details here. The internal geometry of a porous medium is microscopically quantitated by partitioning the pore space into a discrete and well-defined collection of individual pores. Pores are defined as regions of the void space confined by solid surfaces (a.k.a nodal pores, pore bodies, chambers) connected by pore channels (a.k.a pore necks, throats). The pore-throat partitioning is performed by classifying the pore network skeleton into nodal and link points. The geometry of the individual throats is obtained from the cross-sectional area and perimeter of their intersections with planes normal at each link point. Bulk pore interconnectivity is computed from the Euler number $(\chi)$ obtained by 3D connected component analysis. Degree of Anisotropy (DA) and Structure model index (SMI) are computed by characterizing the 
preferential orientational and topological alignment of pores. Geometrical tortuosity is computed as the distribution of the shortest path lengths between all possible skeletal voxel pairs incident on the two faces considered.

Figure 5 shows the pore skeletal network in the central region of the GRF scaffolds. The individual nodes on the skeleton are color coded based on their nearest distance to a solid voxel. Qualitatively, the skeletal networks seem to be highly interconnected. Table 1 summarizes the quantitative results of image-based metrology. The effective pore radius was obtained by finding the radius of a sphere of equivalent volume. The effective throat radius is the radius of a circle of equivalent area. To compute the throat pore radii, the average of two different adjoining pore bodies was computed for each throat. The global porosity deviates from the initial inplane porosity $(60 \%)$ due to dilations introduced by shape-based interpolation.

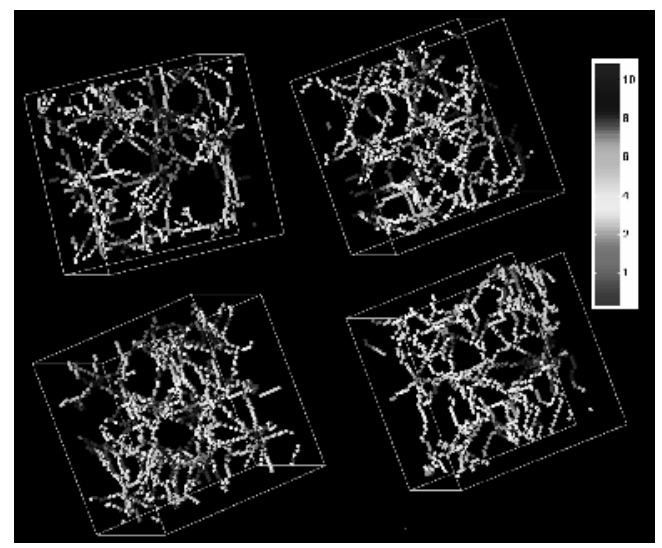

Fig. 5. Qualitative visualization of the skeleton of GRF scaffolds. The medial axis correspond to the central region of the GRF scaffolds modeled respectively (left to right, top to bottom) with Matern, spherical, exponential and rational quadratic covariance functions.

Table 1. Summary of image-based metrology of GRF-scaffolds

\begin{tabular}{|c|c|c|c|c|c|c|c|c|}
\hline GRF & $\begin{array}{c}\phi \\
(\%)\end{array}$ & $\chi$ & $\tau_{\mathrm{Av}}$ & $\begin{array}{l}\text { EPR } \\
(\mu \mathrm{m}) \\
\end{array}$ & $\begin{array}{l}\text { ETR } \\
(\mu \mathrm{m}) \\
\end{array}$ & DA & SMI & $\begin{array}{l}\text { Key: } \\
\phi \text { - porosity }\end{array}$ \\
\hline$C_{M}(1,1)$ & 63 & -6.5 & 1.50 & 382 & 421 & 0.83 & 0.98 & $\chi$ - Euler Number \\
\hline$C_{M}(1,2)$ & 61 & -38.5 & 1.47 & 527 & 510 & 0.73 & 0.55 & $\tau_{\mathrm{Av}}-$ Avg.tortuosity \\
\hline $\mathrm{C}_{\mathrm{S}}(\mathbf{5})$ & 61 & -5.75 & 1.49 & 428 & 472 & 0.79 & 0.79 & EPR - Avg. eff. pore radii \\
\hline $\mathrm{C}_{\mathrm{S}}(\mathbf{1 0})$ & 59 & $\begin{array}{l} \\
21.75 \\
\end{array}$ & 1.54 & 562 & 494 & 0.63 & 0.27 & $\begin{array}{l}\text { ETR - Avg. eff. throat } \\
\text { radii }\end{array}$ \\
\hline$C_{E}(9,15)$ & 62 & $\begin{array}{l}- \\
21.25 \\
\end{array}$ & 1.55 & 560 & 576 & 0.5 & 0.14 & $\begin{array}{l}\text { DA - anisotropy degree. } \\
\text { SMI - struct. model index }\end{array}$ \\
\hline$C_{R}(11,11)$ & 63 & -16.5 & 1.50 & 397 & 438 & 0.68 & 0.66 & \\
\hline
\end{tabular}


The Euler number for all the scaffolds are negative indicating high pore interconnectivity- a characteristic hardly achievable with conventional stochastic scaffolds. The tortuosity for all the GRF scaffolds characterized is within the 1.41-1.78 range considered to be optimal for mass transport through globally interconnected porous space [21]. Effective pore and throat radii are within the ranges required for orthopedic tissue engineering scaffolds [20]. Results for degree of anisotropy shows that all the scaffolds are anisotropic- a favored attribute for multidirectional tissue growth. Non negative values for SMI indicate the absence of concave surfaces and enclosed cavities typically found in suboptimal conventional scaffolds.

\section{GRF Scaffold Fabrication and Mechanical Characterization}

The volumetric binary GRF can be directly converted into surface models suitable for fabrication with rapid prototyping devices. Figure 6 shows the geometric .STL model within a sub region of a GRF scaffold. Multiple copies of the six scaffolds shown in Table 1 were fabricated with a Stereolithography machine (3D Systems, CA). The machine uses a UV laser that is vector scanned over the top of a bath of photopolymerizable liquid polymer material. As polymerization is initiated, the laser beam creates a first solid plastic layer, at, and just below the surface of the bath. This laser polymerization process is repeated to generate subsequent layers by tracing the laser beam along the design boundaries and filling in the $2 \mathrm{D}$ cross-section of the model, layer-by-layer, in steps of 0.02 inches. After building the model, excess resin was removed and cured in a UV oven.

For mechanical characterization, the GRF scaffolds were uniaxially compressed on a Dynamic Mechanical Analyzer (DMA: TA Inst., New Castle, DE). Five specimens for each of the GRF scaffolds were used for the mechanical testing. The specimens were uniaxially compressed with parallel plates by applying a ramp force of $4 \mathrm{~N} / \mathrm{min}$ for 4.5 minutes. Figure 7 compares the linear modulus on the different scaffolds. Preliminary analysis of the results indicates that the mechanical strength adequately meets the requirements of tissue engineering scaffolds [1]. More investigation is needed to link the destructive mechanical testing with the nondestructive image metrology.

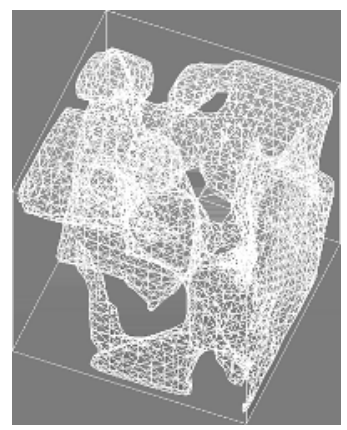

Fig. 6. Polygonization of the GRF scaffold architecture. The wireframe shows a sub region extracted from one of the GRF scaffolds. 


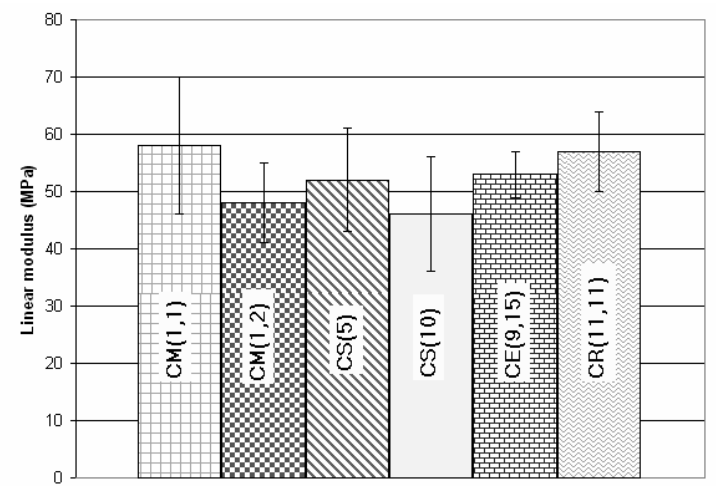

Fig. 7. Linear modulus of GRF scaffolds $(\mathrm{N}=5)$ based on uniaxial compression on DMA under identical loading conditions ( $4 \mathrm{~N} / \mathrm{min}$. ramp force for 4.5 minutes)

\section{Conclusions}

Recognizing the "random walk through the design space" as the critical bottleneck in the status quo of tissue engineering scaffolds, we have proposed a cosmological approach to adapt the randomness in GRF towards modeling optimized and predictable scaffolds and fabricating them with deterministic processes. In conjunction with appropriate biomaterials, the proposed image guided, domain knowledge driven, predicate rich, computer assisted intervention might contribute to the generation of "living" prosthesis that could integrate with the host tissue thereby reducing the need for further surgery or possible implant failure.

\section{References}

1. Yang S., et al., Design of scaffolds for use in Tissue Engineering: Part I Traditional Factors, Tissue Engineering 7(6), (2001), 679-689.

2. Rajagopalan S, Robb RA., Schwarz meets Schwann: Design and fabrication of Tissue Engineering Scaffolds, MICCAI 2005, 794-801.

3. Spalazzi JP., et al., Osteoblast and Chondrocyte interactions during coculture on scaffolds, IEEE Eng. Med \& Biol., 22(5) (2003) 27-34.

4. Coles P, Chiang LY., Characterizing the nonlinear growth of large-scale structure in the universe, Nature, 406 (2000) 376-378.

5. Chiang LY., et al., Return mapping of phases and the analysis of the gravitational clustering hierarchy, Mon. Not. R. Astron. Soc., 337 (2002) 488-494.

6. Berger JO., et al., Objective Bayesian analysis of spatially correlated data, J Am Stat Assoc., 96(456) (2001) 1361-1374.

7. Matern B., Spatial Variation, Second Edition, Springer Verlag, Berlin, 1986.

8. Wackernagel H., Multivariate Geostatistics, Springer Verlag, Berlin, 1995.

9. Yaglom AM., Correlation theory of stationary and related random functions I. Basic results, Springer Verlag, New York, 1987.

10. Teubner M., Level surfaces of Gaussian random fields and microemulsions, Europhys Lett., 14(1991) 403-408. 
11. Knackstedt MA, Robers AP., Morphology and macroscopic properties of conducting polymer blends, Macromolecules, 29 (1996) 1369-1371.

12. -ibid-., Mechanical and transport properties of model foamed solids, J. Mater Sci Lett, 14 (1995) 1357-1359.

13. Press WH., et al., Numerical recipes in C. The art of scientific computing, $2^{\text {nd }}$ Edn, Cambridge University Press, 1992.

14. Kozintsev B., Computations with Gaussian Random Fields, PhD Thesis, University of Maryland, 1999.

15. Rajagopalan S, Robb RA., Assessment of similarity indices to quantify segmentation accuracy of scaffold images for tissue engineering, Proc SPIE 5747:1636-47, Medical Imaging 2005.

16. Dice LR, Measures of the amount of ecologic association between species, Ecology 26 (1945) 297-302.

17. Rajagopalan S, Karwoski RA., Robb RA., Shape based interpolation of porous amd tortuous binary objects, MICCAI 2002, 957-58.

18. Cima LG et al., Tissue engineering by cell transplantation using degradable polymer substrates, J. Biomech Eng. 113 (1991) 143-151.

19. Rajagopalan S, Robb RA., Image based metrology of porous tissue engineering scaffolds, Proc SPIE Vol 6144:540-550, Medical Imaging 2006.

20. Ramanujan S, Pluen A., Diffusion and convection in collagen gels: implications for transport in the tumor interstitium, J Biophys, 83 (2002) 1650-60.

21. Karageorgiou V, Kaplan D., Porosity of 3D biomaterial scaffolds and osteogenesis, Biomaterials, 26(27)(2005) 5474-91. 